\title{
ESTUDO NUTRICIONAL DA CANAFÍSTULA (II): EFICIÊNCIA NUTRICIONAL EM FUNÇÃO DA ADUBAÇÃO COM NITROGÊNIO E FÓSFORO ${ }^{1}$
}

\begin{abstract}
Natália Hilgert de Souza ${ }^{2}$, Marlene Estevão Marchetti ${ }^{3}$, Thiago de Oliveira Carnevali², Diovany Doffinger Ramos ${ }^{4}$, Silvana de Paula Quintão Scalon ${ }^{3}$ e Eulene Francisco da Silva ${ }^{5}$

RESUMO - Objetivou-se, neste trabalho, avaliar o efeito de doses combinadas de $\mathrm{N}$ e $\mathrm{P}$ no conteúdo de nutrientes, bem como na eficiência nutricional de mudas de canafístula (Peltophorum dubium Spreng. Taub.), em Latossolo Vermelho distroférrico. O experimento foi desenvolvido em vasos de $4 \mathrm{dm}^{3}$, com os tratamentos dispostos em fatorial 4x4, sendo quatro doses de $\mathrm{N}$ - fonte ureia $\left(0 ; 20,82 ; 41,64 ; \mathrm{e} 62,46 \mathrm{mg} \mathrm{kg}^{-1} \mathrm{de} \mathrm{N}\right)$ e quatro doses de $\mathrm{P}$ - fonte superfosfato triplo $\left(0 ; 41,72 ; 83,72\right.$; e $\left.125,16 \mathrm{mg} \mathrm{kg}^{-1} \mathrm{de}_{2} \mathrm{O}_{5}\right)$, em delineamento experimental de blocos casualizados com três repetições. As maiores doses de $\mathrm{N}$ e $\mathrm{P}$ proporcionaram maior acúmulo de macronutrientes tanto na parte aérea quanto na raiz, enquanto nos micronutrientes esse efeito foi ocasionado somente pelo $\mathrm{P}$. A espécie apresentou alto requerimento nutricional quanto ao $\mathrm{N}$, utilizou mais eficientemente o P, e o aporte de nutrientes é maior na parte aérea das mudas.
\end{abstract}

Palavras-chave: Peltophorum dubium, Absorção e translocação de nutrientes.

\section{NUTRITION STUDY OF CANAFÍSTULA (II): NUTRITIONAL EFFICIENCY AS A FUNCTION OF NITROGEN AND PHOSPHORUS FERTILIZATION}

\begin{abstract}
The objective of this study was to evaluate the effect of combined levels of $N$ and $P$ in nutrient content and nutritional efficiency in seedlings of canafístula (Peltophorum dubium Spreng. Taub.) on Oxisol. The experiment was carried out in pots of $4 \mathrm{dm}^{3}$, with treatments arranged in $4 x 4$ factorial scheme, four $N$ levels $\left(0,20.82,41.64\right.$ and $\left.62.46 \mathrm{mg} \mathrm{kg}^{-1} \mathrm{~N}\right)$ and four P levels $\left(0,41.72,83.72\right.$ and $125.16 \mathrm{mg} \mathrm{kg}^{-1}$ of $\mathrm{P}_{2} \mathrm{O}_{5}$ in a randomized block design with three replications. The highest levels of $N$ and $P$ showed higher accumulation of nutrients in both the shoot and root while for the micronutrients that effect was caused only by $P$. The species showed high nutritional requirement for $N, P$ used more efficiently and supply of nutrients is greater for shoot seedling.
\end{abstract}

Keywords: Peltophorum dubium, Absorption and nutrient translocation.

\section{INTRODUÇÃO}

Peltophorum dubium (Sprengel) Taubert, conhecida como canafístula ou angico- amarelo, é uma espécie de ampla ocorrência no Brasil, sobretudo em áreas de Cerrado. É uma espécie rústica e de rápido crescimento, podendo atingir $40 \mathrm{~m}$ de altura. Sua madeira é dura, moderadamente pesada e de longa duração (LORENZI, 2002), com alburno róseo-acinzentado e cerne róseo ou avermelhado, recebendo bem o verniz (VENTURIN et al., 1999). Considerada como madeira de qualidade por diversos autores (BACKES et al., 2002; CARVALHO,

\footnotetext{
${ }^{1}$ Recebido em 04.07.2011 aceito para publicação em 04.06.2012.

${ }^{2}$ Universidade Federal da Grande Dourados, Programa de Pós-Graduação em Agronomia. E-mail: <natalia_hilgert@ hotmail.com> e $<$ thiagocarnevali@live.com $>$.

${ }^{3}$ Universidade Federal da Grande Dourados, Faculdade de Ciências Agrárias. E-mail: <marlenemarchetti@ufgd.edu.br >e <silvanascalon@ufgd.edu.br>.

${ }^{4}$ Doutorado em Ciências Agrárias na Universidade Federal da Grande Dourados, UFGD, Brasil. E-mail: <diovany3@ hotmail.com>.

${ }^{5}$ Pesquisadora da Universidade Federal da Grande Dourados, Faculdade de Ciências Agrárias. E-mail: <eulene_silva@yahoo.com.br>.
} 
2003; MATTEI; ROSENTHAL, 2002; RUCHEL et al., 2003), com produção volumétrica já registrada por Carvalho (2003) de 19,60 m ha' ano $^{-1}$.

O conhecimento dos aspectos nutricionais de espécies nativas do Cerrado é ferramenta importante para compreender o estabelecimento destas em solos com baixa disponibilidade nutricional. Os latossolos representam cerca de $46 \%$ dos solos do Cerrado, são altamente intemperizados, com reduzida disponibilidade de nutrientes e alta saturação por alumínio (EBERHARDT et al., 2008). Além disso, apresentam reação ácida, baixa capacidade de troca catiônica e alta capacidade de adsorção aniônica, sendo a fração argila composta principalmente por caulinita e óxidos de Fe e Al. Os baixos teores de matéria orgânica entre 20 e $30 \mathrm{~g} \mathrm{~kg}^{-1}$ resultam em baixo conteúdo de $\mathrm{N}$ potencialmente mineralizável (RESCK et al., 2008). Com isso, a absorção de nutrientes pelas plantas é prejudicada, especialmente tratando-se de nitrogênio $(\mathrm{N})$ e fósforo $(\mathrm{P})$.

A característica comum de deficiência nutricional em solos ácidos impõe restrições ao crescimento da planta (FURTINI NETO et al., 1999). Muitas espécies arbóreas nativas do Cerrado desenvolveram mecanismos de adaptação a essas condições (HARIDASAN, 2000), porém são escassos trabalhos que busquem verificar quais são e de que forma são utilizados tais mecanismos. Do ponto de vista nutricional, a utilização de parâmetros para avaliar a eficiência nutricional, como eficiência de absorção e de uso e translocação de nutrientes, tem sido utilizada.

Alguns trabalhos vêm sendo realizados para obter informações nutricionais com plantas nativas em fase de muda. Em estudo realizado com a canafístula, verificou-se que a espécie é altamente exigente, principalmente em relação a $\mathrm{N}$ e $\mathrm{P}$, assimilando na parte aérea 260,5 e $12,2 \mathrm{mg} \mathrm{kg}^{-1}$ de $\mathrm{Ne} \mathrm{P}$, respectivamente, quando supridas em solução nutritiva completa (VENTURIN et al., 1999). Outras espécies como a grápia (Apuleia leiocarpa) (NICOLOSO et al., 2007) e o ipê-roxo (Tabebuia impetiginosa) (SOUZA et al., 2006) também foram relatadas como exigentes em $\mathrm{N}$ e $\mathrm{P}$, sendo imperativos para o crescimento das mudas.

Quanto ao fornecimento de P, Fernandes et al. (2000) verificaram que a paineira (Chorisia speciosa) apresentou elevada taxa de absorção quando comparada ao jambolão (Syzygium jambolanum) e à aroeirinha (Schinus terenbinthifolius) supridos com altas doses
(0 a $600 \mathrm{mg} \mathrm{kg}^{-1}$ ). Algumas suposições feitas por esses autores são de que a paineira, devido ao melhor ajuste metabólico das frações fosfatadas, pode ser plantada em solos com diferentes níveis de $\mathrm{P}$ e o jambolão, mais exigente do que a aroeirinha, é indicado para solos com maior disponibilidade de P.

Diante da importância do adequado fornecimento de nutrientes às plantas, no que concerne à fase de muda, este estudo objetivou avaliar o efeito de doses combinadas de $\mathrm{N}$ e $\mathrm{P}$ no conteúdo de macro e micronutrientes, bem como na eficiência nutricional de mudas de canafístula, em Latossolo Vermelho distroférrico.

\section{MATERIAL E MÉTODOS}

O experimento foi desenvolvido de março a setembro de 2009, em casa de vegetação, na Universidade Federal da Grande Dourados (UFGD), em Dourados, MS, nas coordenadas de 22 $11^{\circ} 45^{\prime \prime} \mathrm{S}$ e $54^{\circ} 55^{\prime} 18^{\prime \prime} \mathrm{W}$, com altitude de $446 \mathrm{~m}$. O clima é do tipo Cwa mesotérmico úmido, segundo a classificação de Köppen. A precipitação média anual é de 1.500 mm e a temperatura média, de $22^{\circ} \mathrm{C}$.

As sementes de canafístula foram coletadas no Campus da UFGD em julho/2008 e armazenadas em sacos de papel em temperatura ambiente. Em fevereiro/2009, as sementes foram tratadas com acetona P.A., sendo embebidas por $20 \mathrm{~min}$ e posteriormente lavadas em água corrente e secas em papel-toalha (SCALON et al., 2007). Em seguida, foram semeadas em bandejas de poliestireno com substrato agrícola comercial Bioplant $®$ (à base de casca de Pinus, agentes agregantes, vermiculita, fibra de coco e complementos minerais -NPK + micronutrientes) e mantidas em casa de vegetação com sombrite $50 \%$, permanecendo nessas condições por 30 dias, com duas irrigações diárias através do sistema de aspersão.

O solo utilizado foi um Latossolo Vermelho distroférrico (EMBRAPA, 1999) de textura argilosa coletado do horizonte B, nas imediações da universidade, com os seguintes atributos químicos, determinados conforme metodologia de Claessen (1997): pH em água = 4,7; $\mathrm{pH} \mathrm{em} \mathrm{CaCl}_{2}=4,2$; matéria orgânica $=8,2 \mathrm{~g} \mathrm{~kg}^{-1}$; $\mathrm{P}=1,0 \mathrm{mg} \mathrm{dm}^{-3}$ (Mehlich 1); $\mathrm{K}=0,5 \mathrm{mmol}_{\mathrm{c}} \mathrm{dm}^{-3} ; \mathrm{Ca}$ $=4,0 \mathrm{mmol}_{\mathrm{c}} \mathrm{dm}^{-3} ; \mathrm{Mg}=2,0 \mathrm{mmol}_{\mathrm{c}} \mathrm{dm}^{-3} ; \mathrm{Al}=14,1 \mathrm{mmol}_{\mathrm{c}}$ $\mathrm{dm}^{-3} ; \mathrm{H}+\mathrm{Al}=76,0 \mathrm{mmol}_{\mathrm{c}} \mathrm{dm}^{-3} ; \mathrm{SB}=6,5 \mathrm{mmol}_{\mathrm{c}} \mathrm{dm}^{-3}$; $\mathrm{T}=82,5 \mathrm{mmol}_{\mathrm{c}} \mathrm{dm}^{-3}$; e V\% $=7,9$.

Revista Árvore, Viçosa-MG, v.36, n.5, p.803-812, 2012 
Para elevar a saturação por bases a 70\%, utilizou-se calcáriodolomíticocomPRNT 100\%, incorporadomanualmente, 30 dias antes do transplante das mudas. Cada vaso foi revestido internamente com sacos plásticos para evitar a perda de água e de nutrientes pela drenagem e foram mantidos úmidos a 70\% da capacidade de campo. Aos 31 dias após a calagem, foram transplantadas duas mudas de canafístula, por vaso, com aproximadamente $10 \mathrm{~cm}$ de altura.

A unidade experimental foi constituída por vasos com capacidade de $4 \mathrm{~kg}$ de solo, com duas plantas por vaso, sendo dois vasos por parcela. O delineamento experimental foi em blocos casualizados com três repetições, com os tratamentos arranjados em esquema fatorial 4 x 4, com quatro doses de $\mathrm{N}(0 ; 20,82 ; 41,64$; e $62,46 \mathrm{mg} \mathrm{kg}^{-1} \mathrm{de} \mathrm{N}$, correspondentes a $0,50,100$ e $\left.150 \mathrm{~kg} \mathrm{ha}^{-1}\right)$ e quatro doses de $\mathrm{P}(0 ; 41,72 ; 83,44$; e $125,16 \mathrm{mg} \mathrm{kg}^{-1} \mathrm{de}_{2} \mathrm{O}_{5}$, correspondentes a 0,100 , 200 e $300 \mathrm{~kg} \mathrm{ha}^{-1}$ ), utilizando como fonte de $\mathrm{N}$ a ureia e a fonte de $\mathrm{P}$, o superfosfato triplo. Os cálculos de adubação foram realizados considerando-se a camada de $20 \mathrm{~cm}$ de solo e densidade de $1,2 \mathrm{~g} \mathrm{~cm}^{-3}$.

A adubação complementar constituiu-se de $60 \mathrm{mg}$ $\mathrm{kg}^{-1} \mathrm{~K}_{2} \mathrm{O}$, tendo como fonte o $\mathrm{KCl}$ e $150 \mathrm{mg} \mathrm{kg}^{-1}$ do formulado comercial de micronutrientes FTE-BR12. A adubação nitrogenada foi parcelada, sendo um terço no transplante, juntamente com $\mathrm{P}, \mathrm{K}$ e micronutrientes, e o restante 30 dias após o transplante (DAT).

Aos 170 DAT, as plantas foram coletadas, separadas em parte aérea e raiz, procedendo-se à secagem do material em estufa de circulação forçada de ar a $60^{\circ}$ $\pm 5^{\circ} \mathrm{C}$. Após a obtenção de massa constante, o material foi pesado e moído para determinação dos teores de macro e micronutrientes (MALAVOLTA et al., 1997). A partir desses teores, determinou-se o conteúdo dos nutrientes com base na massa seca.

A partir da massa seca e do conteúdo dos nutrientes na planta, foram calculados os seguintes índices de eficiência nutricional:

Eficiência de absorcão $\left(\mathrm{mg} \mathrm{g}^{-1}\right)=$ $\underline{\text { (conteúdo total do nutriente na planta) }}$ (SWIADER et al., 1994) (massa seca de raízes)

Eficiência de uso $\left(\mathrm{g}^{2} \mathrm{mg}^{-1}\right)=$ (massa seca total produzida) $^{2}$

$\overline{\text { (conteúdo total do nutriente na planta ) }}$

(SIDDIQI; GLASS, 1981)
Eficiência de translocação

$(\%)=\left[\frac{(\text { conteúdo do nutriente na parte aérea })}{\text { conteúdo total do nutriente na planta })}\right] .100$

(ABICHEQUER; BOHNEN, 1998)

Os dados foram submetidos à análise de variância pelo teste $\mathrm{F}$ a $5 \%$ de probabilidade e os fatores significativos, analisados por meio da análise de regressão, utilizando o programa computacional SAEG 9.0 (RIBEIRO JÚNIOR, 2001) e ajustando os gráficos por meio do programa Sigma Plot 9.0.

\section{RESULTADOS}

O conteúdo de macronutrientes foi influenciado significativamente pelas doses de $\mathrm{N}$ e $\mathrm{P}$, tanto na parte aérea quanto na raiz, sendo os maiores valores obtidos com as maiores doses dos nutrientes ou próximas a estas (Figura 1). Os valores máximos para o conteúdo de macronutrientes encontram-se dispostos na Tabela 1. Não foi possível ajustar um modelo matemático de regressão adequado ao conteúdo de $\mathrm{Ca}$ e $\mathrm{Mg}$ na raiz da canafístula. Assim, apresentaram-se as médias dos tratamentos.

Houve efeito significativo das doses de $\mathrm{N}$ e P sobre a eficiência de absorção (Ea) dos nutrientes avaliados, porém não foi possível ajustar nenhum modelo de regressão, tanto para macro quanto para micronutrientes. Os valores médios da Ea de macronutrientes, em mg g-1, foram de 138,73 $\mathrm{Ea}(\mathrm{N})$; 6,35 $\mathrm{Ea}(\mathrm{P}) ; 18,34 \mathrm{Ea}(\mathrm{K}) ; 36,62$ $\mathrm{Ea}(\mathrm{Ca})$; e 9, $15 \mathrm{Ea}(\mathrm{Mg})$.

A adubação aumentou a eficiência de uso (Eu) de macronutrientes (Tabela 2). O P foi o nutriente mais eficientemente utilizado pelas mudas de canafístula e, com a utilização das maiores doses de $\mathrm{N}$ e $\mathrm{P}$, seu valor alcançou $4,45 \mathrm{~g}^{2} \mathrm{mg}^{-1}$, seguido do $\operatorname{Mg}\left(3,54 \mathrm{~g}^{2} \mathrm{mg}^{-1}\right), \mathrm{N}\left(0,204 \mathrm{~g}^{2} \mathrm{mg}^{-1}\right), \mathrm{K}\left(1,42 \mathrm{~g}^{2} \mathrm{mg}^{-1}\right) \mathrm{e}$ $\mathrm{Ca}\left(0,88 \mathrm{~g}^{2} \mathrm{mg}^{-1}\right)$.

Quanto à eficiência de translocação (Et) de macronutrientes, verificou-se interação significativa entre as dose de $\mathrm{N}$ e $\mathrm{P}$, porém a falta de ajuste significativo impediu a escolha de modelo de regressão. Assim, o percentual médio de translocação dos macronutrientes para parte aérea das mudas de canafístula foi de 78,0\% do N, $84,0 \%$ do P, $55,4 \%$ do K, $92,1 \%$ do Ca e $84,0 \%$ do $\mathrm{Mg}$.

Revista Árvore, Viçosa-MG, v.36, n.5, p.803-812, 2012 

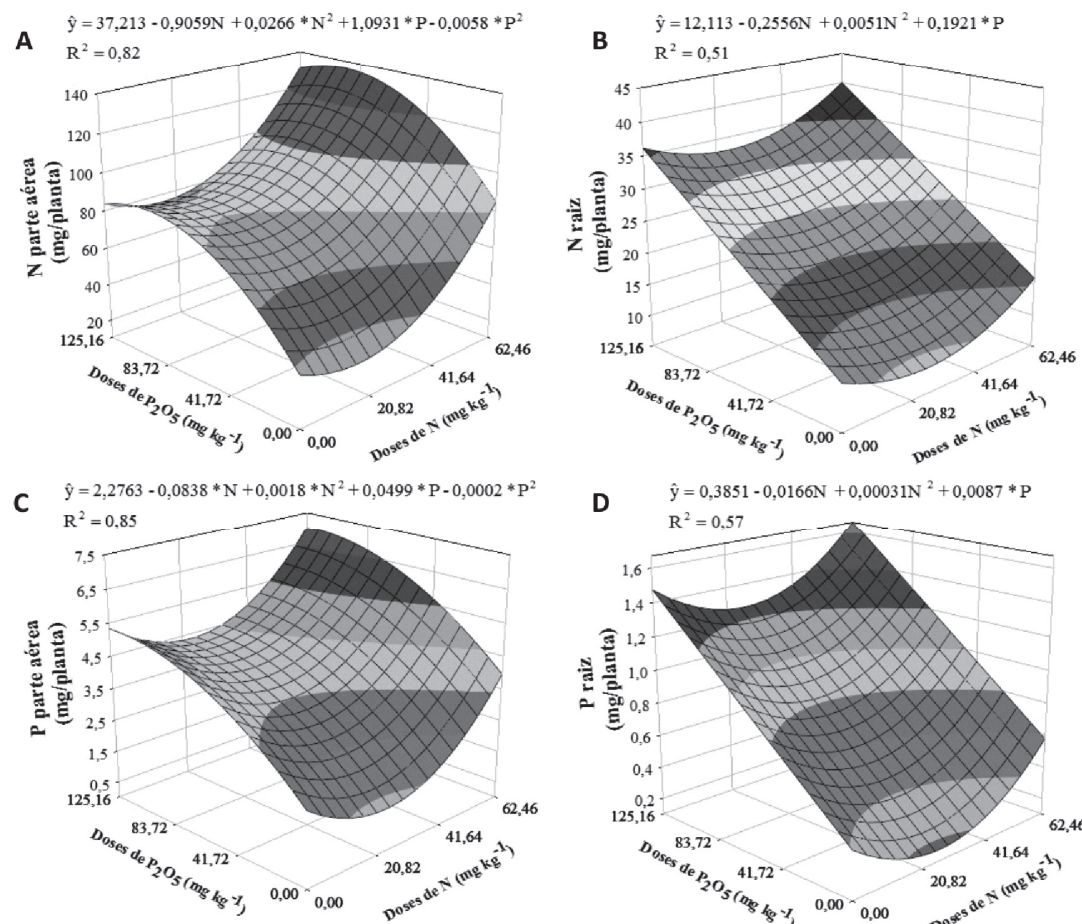

D $\hat{\mathrm{y}}=0,3851-0,0166 \mathrm{~N}+0,00031 \mathrm{~N}^{2}+0,0087 * \mathrm{P}$
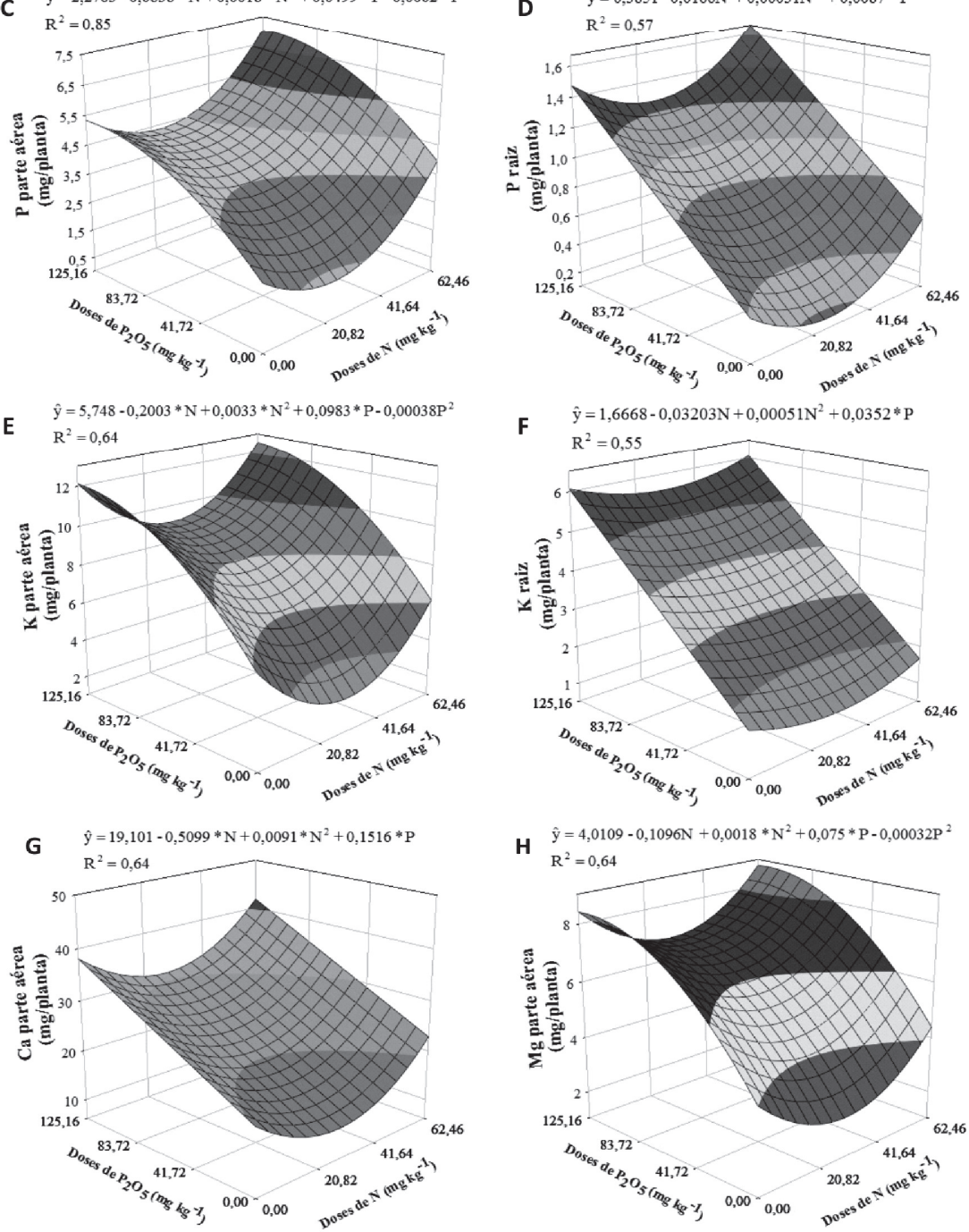

$\hat{\mathrm{y}}=4,0109-0,1096 \mathrm{~N}+0,0018 * \mathrm{~N}^{2}+0,075 * \mathrm{P}-0,00032 \mathrm{P}^{2}$

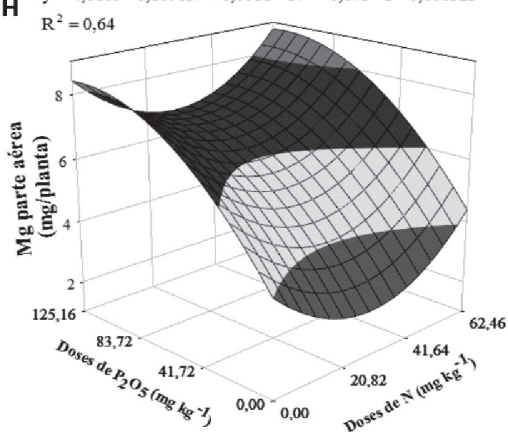

Figura 1 - Conteúdo de macronutrientes na parte aérea (A, C, E, G e H) e na raiz (B, D e F) de mudas de canafístula aos 170 DAT, em função da adubação fosfatada e nitrogenada. UFGD, Dourados, 2009.

Figure 1 - Content of nutrients in shoots $(A, C, E, G$ e H) and roots $(B, D e F)$ of seedlings canafistula at 170 DAT as a function of phosphorus and nitrogen fertilization. UFGD, Dourados, 2009.

Revista Árvore, Viçosa-MG, v.36, n.5, p.803-812, 2012 
Tabela 1 - Conteúdo de macro e micronutrientes em mudas de canafístula aos 170 DAT, em função da adubação fosfatada e nitrogenada. UFGD, Dourados, 2009.

Table 1 - Content of macro and micronutrients for seedlings canafístula at 170 DAT as a function of phosphorus and nitrogen fertilization. UFGD, Dourados, 2009.

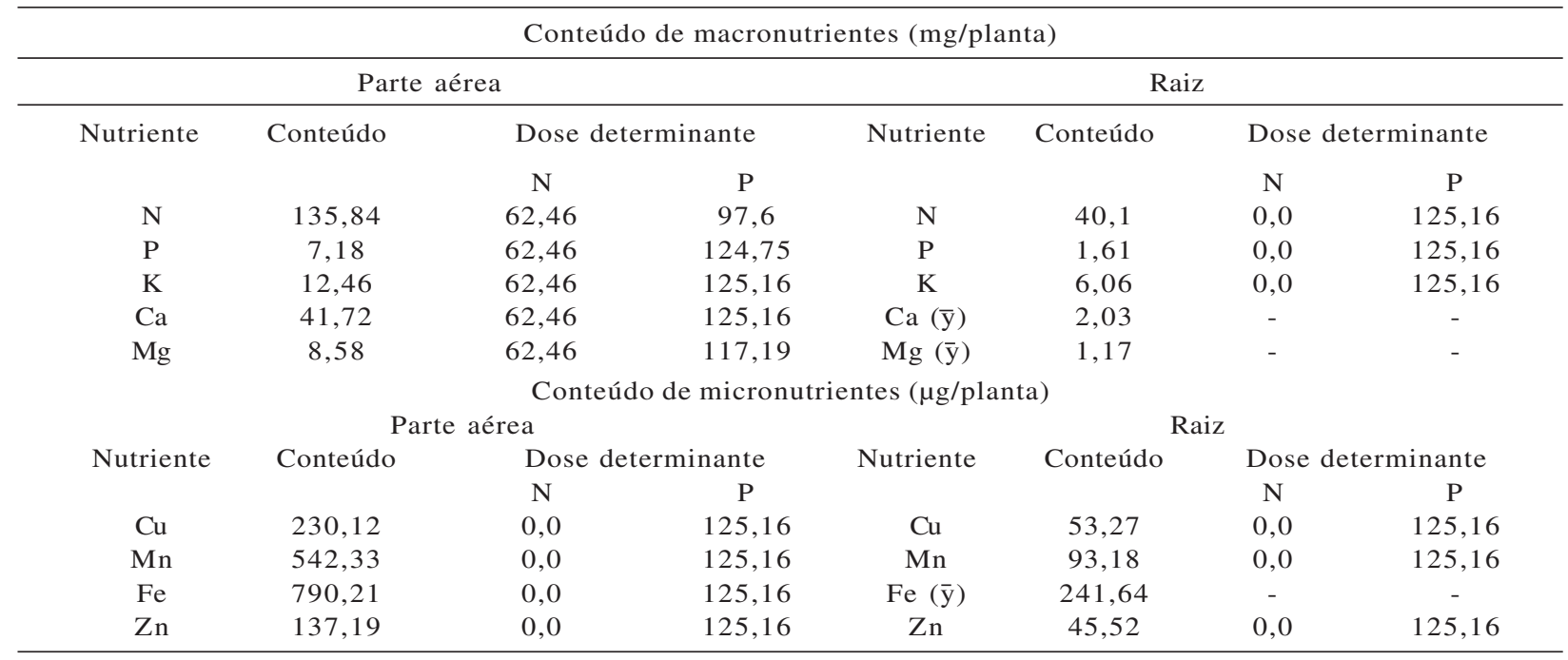

Tabela 2 - Eficiência no uso (Eu) de macronutrientes em mudas de canafístula aos 170 DAT, em função da adubação fosfatada e nitrogenada. UFGD, Dourados, 2009.

Table 2 - Use efficiency of macronutrients for seedlings canafístula at 170 DAT as a function of phosphorus and nitrogen fertilization. UFGD, Dourados, 2009.

\begin{tabular}{ccc}
\hline Eficiência de uso & Equação de regressão para Ea & $\mathrm{R}^{2}$ \\
\hline $\operatorname{Eu}(\mathrm{N})$ & $\hat{\mathrm{y}}=0,1072-0,0051 * \mathrm{~N}+0,000078^{*} \mathrm{~N}^{2}+0,0019 * \mathrm{P}-0,000016 \mathrm{NP}$ & 0,81 \\
$\mathrm{Eu}(\mathrm{P})$ & $\hat{\mathrm{y}}=2,5819-0,0826^{*} \mathrm{~N}+0,00105 * \mathrm{~N}^{2}+0,0234 * \mathrm{P}$ & 0,65 \\
$\mathrm{Eu}(\mathrm{K})$ & $\hat{\mathrm{y}}=0,6616-0,0264 * \mathrm{~N}+0,00043 * \mathrm{~N}^{2}+0,0157 * \mathrm{P}-0,00008 * \mathrm{P}^{2}$ & 0,77 \\
$\mathrm{Eu}(\mathrm{Ca})$ & $\hat{\mathrm{y}}=0,3985-0,0162 * \mathrm{~N}+0,00024 * \mathrm{~N}^{2}+0,00445 * \mathrm{P}$ & 0,73 \\
$\mathrm{Eu}(\mathrm{Mg})$ & $\hat{\mathrm{y}}=1,519-0,0621 * \mathrm{~N}+0,00095 * \mathrm{~N}^{2}+0,0175 * \mathrm{P}$ & 0,76 \\
\hline
\end{tabular}

* Coeficientes da regressão significativos a $5 \%$ de probabilidade.

O conteúdo de micronutrientes, tanto na parte aérea quanto na raiz de mudas de canafístula, foi afetado negativamente pelo incremento nas doses de N (Figura 2). No entanto, os valores máximos foram obtidos com as maiores doses de P. Os valores máximos, com suas doses determinantes, encontram-se na Tabela 1. Não houve ajuste de modelo de regressão para o conteúdo de Fe na raiz, sendo seu valor médio obtido de 241,64 $\mu \mathrm{g} /$ planta.

Devido à falta de ajuste significativo, os valores médios da eficiência de absorção de micronutrientes

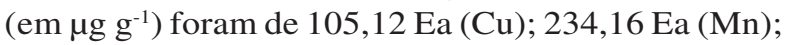
$638,122 \mathrm{Ea}(\mathrm{Fe})$; e 72,9 Ea (Zn). Da mesma forma, o efeito das doses de $\mathrm{N}$ e $\mathrm{P}$ sobre a eficiência no uso de micronutrientes foi significativo, porém não houve ajuste de modelo de regressão. Os valores médios, em mg ${ }^{2} \mathrm{mg}-1$, foram $0,17 \mathrm{Eu}(\mathrm{Cu}), 0,07 \mathrm{Eu}(\mathrm{Mn}), 0,03 \mathrm{EU}(\mathrm{Fe})$ e 0,253 $\mathrm{Eu}(\mathrm{Zn})$.

A eficiência de translocação (Et) de micronutrientes também foi influenciada pela interação entre as doses de $\mathrm{N}$ e P, indicando maior aquisição pela aérea das mudas. As equações de regressão ajustadas encontram-se na Tabela 3. Utilizando-se as maiores doses de $\mathrm{N}$ e P, a Et máxima, em porcentagem, foi 83,8 (Cu); 86,8 (Mn); 80,6 (Zn); e 68,9 (Fe). Houve ponto de mínimo nas doses de $\mathrm{N}$ variando de 31,38 a $32,42 \mathrm{mg} \mathrm{kg}^{-1}$, em todos os 

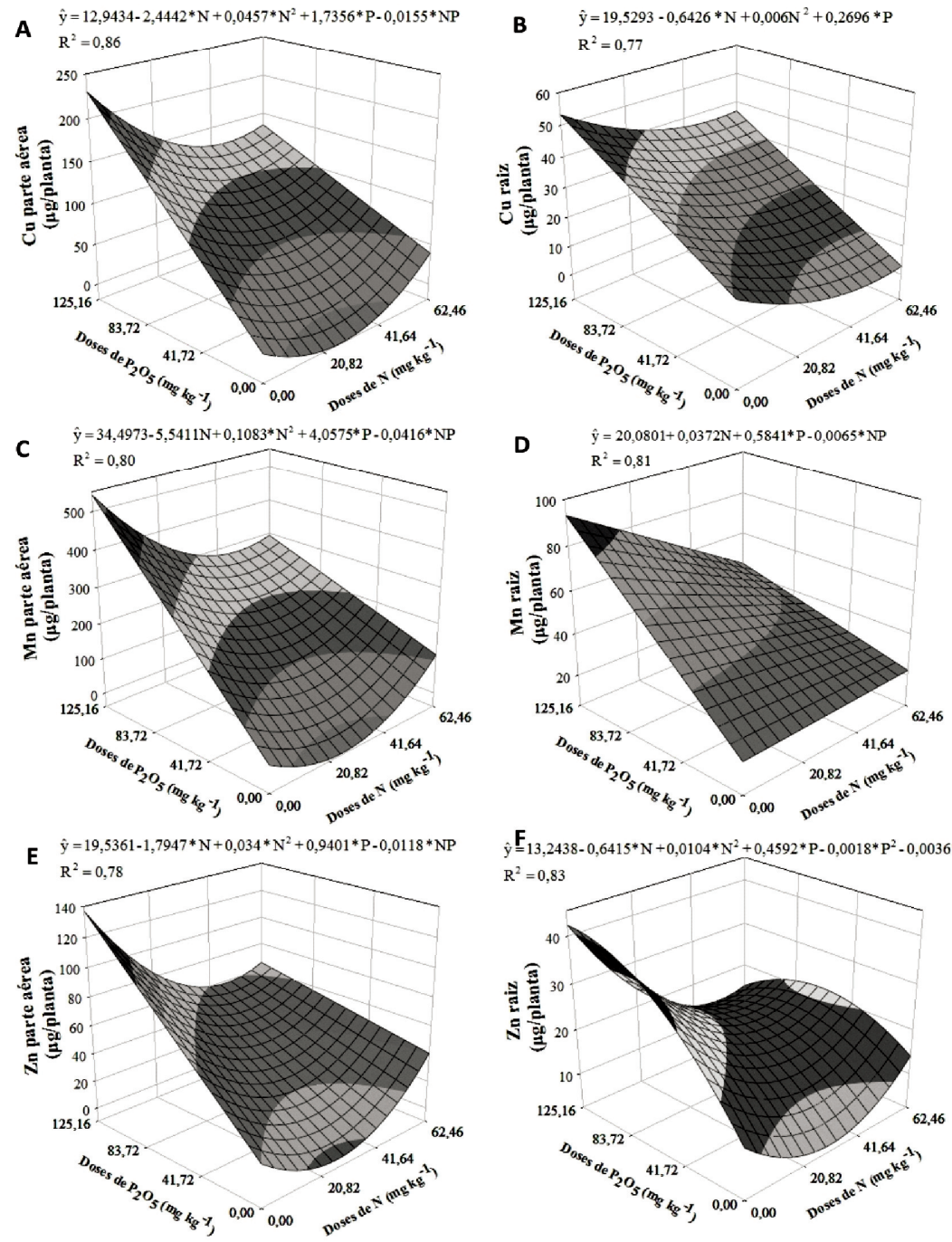

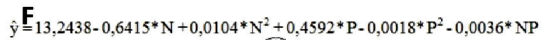
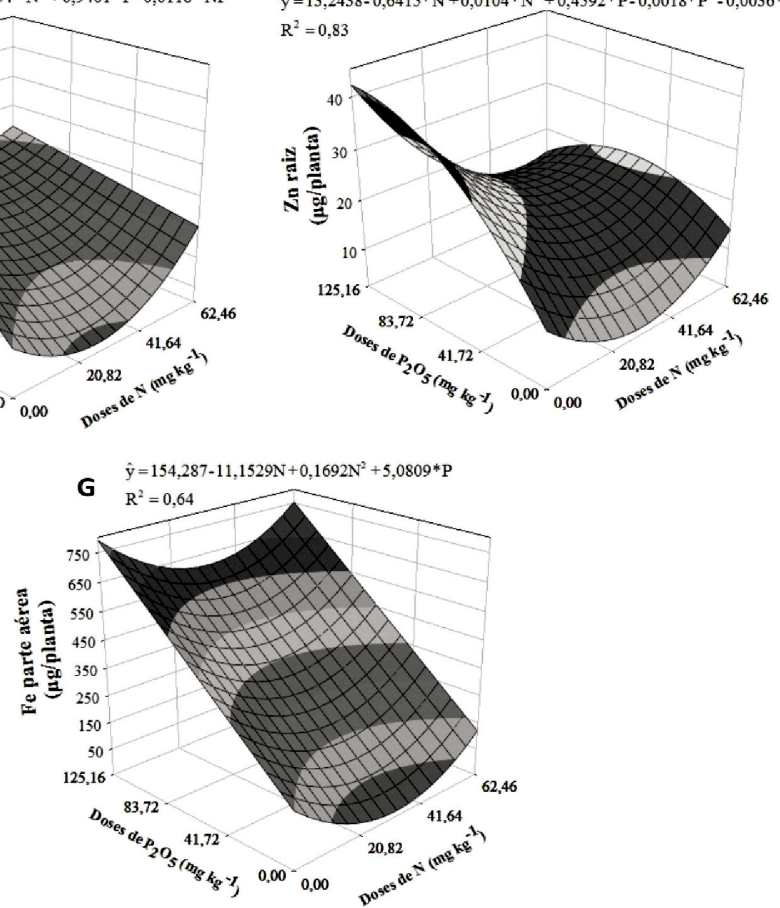

Figura 2 - Conteúdo de micronutrientes na parte aérea (A, C, E e G) e na raiz (B, D e F) de mudas de canafístula aos 170 DAT, em função da adubação fosfatada e nitrogenada. UFGD, Dourados, 2009.

Figure 2 - Content of micronutrients in shoots $(A, C, E$ e $G)$ and roots $(B, D$ e F) of seedlings canafistula at 170 DAT as a function of phosphorus and nitrogen fertilization. UFGD, Dourados, 2009.

Revista Árvore, Viçosa-MG, v.36, n.5, p.803-812, 2012 
Tabela 3 - Eficiência de translocação de micronutrientes (Et) em mudas de canafístula aos 170 DAT, em função da adubação fosfatada e nitrogenada. UFGD, Dourados, 2009.

Table 3 - Translocation efficiency of micronutrients for seedlings canafístula at 170 DAT as a function of phosphorus and nitrogen fertilization. UFGD, Dourados, 2009.

\begin{tabular}{ccc}
\hline Eficiência de translocação & Equação de regressão para Et & $\mathrm{R}^{2}$ \\
\hline $\operatorname{Et}(\mathrm{Cu})$ & $\hat{\mathrm{y}}=60,3-0,775 * \mathrm{~N}+0,012 * \mathrm{~N}^{2}+0,451 * \mathrm{P}-0,002 * \mathrm{P}^{2}$ & 0,76 \\
$\operatorname{Et}(\mathrm{Mn})$ & $\hat{\mathrm{y}}=66,16-0,816 * \mathrm{~N}+0,013 * \mathrm{~N}^{2}+0,457 * \mathrm{P}-0,002 * \mathrm{P}^{2}$ & 0,74 \\
$\operatorname{Et}(\mathrm{Zn})$ & $\hat{\mathrm{y}}=58,832-0,841 * \mathrm{~N}+0,013 * \mathrm{~N}^{2}+0,396 * \mathrm{P}-0,0018 * \mathrm{P}^{2}$ & 0,75 \\
$\mathrm{Et}(\mathrm{Fe})$ & $\hat{\mathrm{y}}=44,454-0,888 * \mathrm{~N}+0,014 * \mathrm{~N}^{2}+0,465 * \mathrm{P}-0,002 * \mathrm{P}^{2}$ & 0,80 \\
\hline
\end{tabular}

* Coeficientes de regressão significativos a $5 \%$ de probabilidade.

micronutrientes. De posse desses dados, verificou-se que a utilização da maior dose de $\mathrm{N}$ proporciona efeito semelhante na translocação de micronutrientes, em comparação com o efeito isolado do P.

\section{DISCUSSÃO}

Estudos sobre nutrição mineral de espécies arbóreas nativas demonstram que estas respondem positivamente à fertilização, principalmente em relação ao nitrogênio e fósforo. Utilizando a técnica do elemento faltante na nutrição mineral de $P$. dubium, Venturin et al. (1999) verificaram que a absorção de nutrientes foi mais prejudicada quando da omissão de $\mathrm{N}$ e $\mathrm{P}$, comparando-se à testemunha. No tratamento completo, aos 96 DAT, utilizando as doses de $150 \mathrm{mg} \mathrm{kg}^{-1} \mathrm{de} \mathrm{Ne} 120 \mathrm{mg} \mathrm{kg}^{-1} \mathrm{de}$ $\mathrm{P}$, o conteúdo de macronutrientes ( $\mathrm{mg} /$ planta) na parte aérea das mudas foi 260,3 (N), 12,2 (P), 87,8 (K), 74,9 (Ca) e 16,4 (Mg). Comparando com esta pesquisa, ressalta-se que as diferenças metodológicas como tratamentos, tipo de solo e tempo antecedente ao transplante possivelmente contribuíram para as diferenças nos conteúdos de macronutrientes para a mesma espécie.

Em mudas de Carapa guianensis, após seis meses de cultivo sob doses crescentes de P, Neves et al. (2004) observaram que os elementos em estudo que mais se acumularam na parte aérea (mg/planta) seguem a seguinte ordem decrescente: N (254,3); Ca (133,5); K (106,5); $\mathrm{Mg}(17,9) ; \mathrm{S}(16,3)$; e P $(6,4)$, esses valores foram obtidos com doses de $\mathrm{P}$ variando de 241 a $287,5 \mathrm{mg} \mathrm{dm}^{-3}$.

A absorção de nutrientes pela planta varia durante o ciclo de desenvolvimento em função da quantidade de raízes e da taxa de absorção por unidade de peso de raiz. Normalmente, essa quantidade aumenta progressivamente durante o período de crescimento, fase em que a planta ganha massa. $\mathrm{O}$ alto requerimento nutricional quanto ao nitrogênio em fase de muda é esperado, pois durante o crescimento vegetativo o $\mathrm{N}$ é rapidamente absorvido pelas raízes e transportado para as folhas (BREDEMEIER; MUNDSTOCK, 2000).

Segundo Silva et al. (2006), nem sempre o maior acúmulo de determinado nutriente significa maior eficiência no seu uso. Considerando a ordem de acúmulo de nutrientes nas mudas, o $\mathrm{P}$ foi o nutriente com menor conteúdo, no entanto foi o mais eficaz em produzir massa seca. O P é citado por muitos autores (NICOLOSO et al., 2007; SOUZA et al., 2006; SOUZA et al., 2010, entre outros) como determinante no crescimento das plantas, uma vez que tem considerável importância no metabolismo do carbono, bem como na formação de açúcares fosfatados. Ao contrário do $\mathrm{P}$, o Ca apresentou elevado conteúdo na planta, porém baixa eficiência de uso. Horst et al. (1992) sugeriram a inativação de Ca devido à ligação ou precipitação na forma de oxalato ou fosfato de cálcio, como causa da baixa eficiência de utilização do nutriente.

A translocação de nutrientes em folhas durante a senescência tem sido intensivamente estudada, no entanto ainda são escassos estudos sobre a translocação de nutrientes nos diferentes compartimentos da planta (LACLAU et al., 2001). A maioria dos trabalhos desta natureza vem sendo desenvolvida com o intuito de verificar o comportamento de algumas espécies arbóreas diante das condições de toxidez por metal pesado, como os de Fernandes et al. (2007) e Paiva et al. (2003).

Quando da ausência de adubação, houve maior acúmulo de Cu na raiz da canafístula (19,53 $\mu \mathrm{g} /$ planta) em relação à parte aérea $(12,94 \mu \mathrm{g} /$ planta $)$. Tal resultado indica que tanto a absorção quanto a translocação de $\mathrm{Cu}$ foram favorecidas em relação à adubação, principalmente ao P. Silva et al. (2011), estudando a

Revista Árvore, Viçosa-MG, v.36, n.5, p.803-812, 2012 
tolerância de três espécies florestais nativas ao excesso de $\mathrm{Cu}$ em Argissolo Vermelho-Amarelo distrófico, verificaram que a espécie $P$. dubium apresenta tendência de armazenamento de $\mathrm{Cu}$ nas raízes e baixa translocação para a parte aérea. Sem a adição de $\mathrm{Cu}$ ao solo, o teor na parte aérea e na raiz foi de 11,57 e 43,37 $\mathrm{mg} \mathrm{kg}^{-1}$, respectivamente.

Não foi observado efeito antagônico do P na absorção do Zn, assim como relatado por autores como Malavolta et al. (1997) e Epstein e Bloom (2006); pelo contrário, a máxima dose de $\mathrm{P}$ aumentou em $86 \%$ a absorção de $\mathrm{Zn}$ na parte aérea e em $71 \%$ na raiz, em relação à não aplicação de P. Utilizando a técnica do elemento faltante na avaliação nutricional de $P$. dubium, Venturin et al. (1999) obtiveram conteúdo de Zn na parte aérea de 1.527,47 $\mu \mathrm{g} /$ planta referente ao tratamento completo, 15,67 $\mu \mathrm{g} /$ planta com a testemunha, $48,12 \mu \mathrm{g} /$ planta com a omissão de $\mathrm{N}$ e 102,9 $\mu \mathrm{g} /$ planta com omissão de P. Através desses dados, observou-se que a capacidade de resposta à absorção de Zn pela canafístula foi variável e sofreu influência direta da interação entre outros nutrientes, principalmente em relação ao $\mathrm{N}$ e ao $\mathrm{P}$.

Entre os micronutrientes, o Fe e o Mn foram os que mais se acumularam nos tecidos das mudas de canafístula, sobretudo na parte aérea. O elevado nível de translocação de metais para a parte aérea pode indicar baixa tolerância a esses elementos, pois, uma vez dentro das células vegetais, sistemas importantes para a sobrevivência e desenvolvimento da espécie, como funções dos fotossistemas I e II, podem ser afetados (P ̈̈TSIKKÄ et al., 2002).

A eficiência nutricional das espécies apresenta controle genético (PAULA et al., 2003; TOMAZ et al., 2003) e pode fornecer subsídios a programas de melhoramento genético, os quais poderão selecionar espécies com maior capacidade de absorver e assimilar nutrientes quando em condições limitantes. Espécies nativas possuem alto grau de variabilidade genética e, quando submetidas a condições experimentais, podem não responder de acordo com o esperado. Neste trabalho, a falta de ajuste na maioria dos nutrientes em relação à eficiência de absorção, de uso e translocação pelas mudas de canafístula pode indicar o efeito da variabilidade genética da canafístula. Assim, sugere-se que novas pesquisas sejam realizadas no âmbito interdisciplinar, a fim de obter recursos genéticos de grande valor, seja para sistemas de produção, seja para programas de melhoramento genético.

Revista Árvore, Viçosa-MG, v.36, n.5, p.803-812, 2012

\section{CONCLUSÕES}

A canafístula apresentou alto requerimento nutricional quanto ao $\mathrm{N}$, utilizou mais eficientemente o P e teve maior translocação de nutrientes para a parte aérea.

A ordem de acúmulo dos nutrientes foi de $\mathrm{N}>\mathrm{Ca}>\mathrm{K}>\mathrm{Mg}>\mathrm{P}>\mathrm{Fe}>\mathrm{Mn}>\mathrm{Cu}>\mathrm{Zn}$, entretanto na raiz foi de $\mathrm{N}>\mathrm{K}>\mathrm{Ca}>\mathrm{P}>\mathrm{Mg}>\mathrm{Fe}>\mathrm{Mn}>\mathrm{Cu}>\mathrm{Zn}$. A ordem decrescente de acúmulo de nutrientes na parte aérea foi de $\mathrm{N}>\mathrm{Ca}>\mathrm{K}>\mathrm{Mg}>\mathrm{P}>\mathrm{Fe}>\mathrm{Mn}>\mathrm{Cu}>\mathrm{Zn}$ e na raiz, $\mathrm{N}>\mathrm{K}>\mathrm{Ca}>\mathrm{P}>\mathrm{Mg}>\mathrm{Fe}>\mathrm{Mn}>\mathrm{Cu}>\mathrm{Zn}$.

\section{AGRADECIMENTOS}

À Fundação de Apoio ao Desenvolvimento do Ensino, Ciência e Tecnologia do Estado de Mato Grosso do Sul, pela concessão de bolsa de estudos e pelo apoio financeiro.

\section{REFERÊNCIAS}

ABICHEQUER, A. D.; BOHNEN, H. Eficiência de absorção, translocação e utilização de fósforo por variedades de trigo. Revista Brasileira de Ciência do Solo, v.22, n.1, p.21-26, 1998.

BACKES, P.; IRGANG, B. Árvores do Sul: guia de identificação \& interesse ecológico. Porto Alegre: Pallotti, 2002. 326p.

BREDEMEIER, C.; MUNDSTOCK, C.M. Regulação da absorção e assimilação do nitrogênio nas plantas. Ciência Rural, v.30, n.2, p.365-372, 2000.

\section{CARVALHO, P.E.R. Espécies arbóreas}

brasileiras. Brasília: Embrapa Informação Tecnológica; Colombo: Embrapa Florestas, 2003. v.2. 1036 p.

CLAESSEN, M. E. C. (Org.). Manual de métodos de análises de solo. 2.ed. Rio de Janeiro: EMBRAPA-CNPS, 1997. 212p.

(Documentos, 1).

EBERHARDT, D. N. et al. Influência da granulometria e da mineralogia sobre a retenção do fósforo em latossolos sob pastagens no cerrado. Revista Brasileira de Ciência do Solo, v.32, n.3, p.1009-1016, 2008. 
EMPRESA BRASILEIRA DE PESQUISA

AGROPECUÁRIA - EMBRAPA. Centro Nacional de Pesquisa de Solos. Sistema brasileiro de classificação de solos. Brasília: 1999. 412p.

EPSTEIN, E.; BLOOM, A. J. Nutrição mineral de plantas: princípios e perspectivas. Londrina: Planta, 2006. 402p.

FERNANDES, A. R. et al. Crescimento e absorção de nutrientes por mudas de freijó (Cordia goeldiana Huber) em função de doses de fósforo e de zinco. Revista Árvore, v.31, n.4, p.599-608, 2007.

FERNANDES, L. A. et al. Crescimento inicial, níveis críticos de fósforo e frações fosfatadas em espécies florestais. Pesquisa

Agropecuária Brasileira, v.35, n.6, p.1191-1198, 2000.

FURTINI NETO, A. E. et al. Acidez do solo, crescimento e nutrição mineral de algumas espécies arbóreas, na fase de muda. Cerne, v.5, n.2, p.1-12, 1999.

HARIDASAN, M. Nutrição mineral de plantas nativas do cerrado. Revista Brasileira de Fisiologia Vegetal, v. 12, n.1, p.54-64, 2000.

HORST, W. J.; CURRLE, C.; WISSEMEIER, A. H. Differences in calcium efficiency between cowpea (Vigna unguiculata (L.) Walp.) cultivars. Plant Soil, v.146, n.1, p.45-54, 1992.

LACLAU, J. P. et al. Dynamics of nutrient translocation in stemwood across an age series of a Eucalyptus Hybrid. Annals of Botany, v.88, p.1079-1092, 2001.

LORENZI, H. Árvores brasileiras. 4.ed. Nova Odessa: Instituto Plantarum, 2002. v.2. 202p.

MALAVOLTA, E.; VITTI, G. C.; OLIVEIRA, S. A. Avaliação do estado nutricional das plantas: princípios e aplicações. Piracicaba: Associação Brasileira para a Pesquisa da Avaliação da Potassa e do Fosfato, 1997. 319p.

MARSCHNER, H. Mineral nutrition of higher plants. 2.ed. San Diego: Academic, 1997. 889p.
MATTEI, V. L.; ROSENTHAL, M. D. Semeadura direta de canafístula (Peltrophorum dubium (Spreng.) taub.) no enriquecimento de capoeiras. Revista Árvore, v.26, n.6, p.649-654, 2002.

NEVES, O. S. C. et al. Crescimento, produção de massa seca e acúmulo de N, P, K, Ca, Mg e $\mathrm{S}$ na parte aérea de mudas de andiroba (Carapa guianensis Aubl.) cultivadas em solo de várzea, em função de diferentes doses de fósforo. Revista Árvore, v.28, n.3, p.343-349, 2004.

NICOLOSO, F. T. et al. Exigências nutricionais da grápia em Argissolo Vermelho distrófico arênico: (II) Efeito da adubação NPK no teor de nutrientes nos tecidos. Ciência Rural, v.37, n.2, p.372-380, 2007.

PAIVA, H. N. et al. Teor, conteúdo e índice de translocação de nutrientes em mudas de cedro (Cedrela fissilis Vell.) submetidas a doses crescentes de zinco. Ciência Florestal, v.13, n.1, p.1-10, 2003.

PÄTSIKKÄ, E. et al. Excess cooper predisposes photosystem II to photoinhibition in vivo by outcompeting iron and causing decrease in leaf chlorophyll. Plant Physioloy, v.129, p.13591367, 2002.

PAULA, R. C. et al. Controle genético da eficiência de utilização de fósforo em famílias de meios-irmãos deEucalyptus grandis, em casa de vegetação. Revista. Árvore, v.27, n.1, p.15-23, 2003.

RESCK, D. V. S. et al. Dinâmica da matéria orgânica no Cerrado. In: SANTOS, G.A. et al. (Eds). Fundamentos da matéria orgânica do solo: ecossistemas tropicais \& subtropicais. 2.ed. Porto Alegre: Metrópole, 2008. 645p.

RIBEIRO JÚNIOR, J. I. Análise estatística no SAEG. Viçosa: Universidade Federal de Viçosa, 2001. 301p.

RUCHEL, A. R. et al. Evolução do uso e valorização das espécies madeiráveis da floresta estacional decidual do alto Uruguai. Ciência Florestal, v.13, n.1, p.153-166, 2003.

Revista Árvore, Viçosa-MG, v.36, n.5, p.803-812, 2012 
SCALON, S. P. Q. et al. Potencial germinativo de sementes de Dimorphandra mollis Benth. armazenamento, tratamentos pré-germinativos e temperatura de incubação. Cerne, v.13, n.3, p.321-328, 2007.

SIDDIQI, M.; GLASS, A. D. M. Utilization index: a modified approach to the estimation and comparison of nutrient utilization efficiency in plants. Journal of Plant Nutrition, v.4, p.289-302, 1981.

SILVA, C. E. M. et al. Eficiência no uso dos nutrientes por espécies pioneiras crescidas em pastagens degradadas na Amazônia central. Acta Amazônica, v.36, n.4, p.503-512, 2006.

SILVA, R. F. et al. Comportamento de Peltophorum dubium (Spreng.) Taub., Parapiptadenia rigida (Benth.) Brenan e Enterolobium contortisiliquum (Vell.) Morong cultivadas em solo contaminado com cobre. Ciência Florestal, v.21, n.1, p.103-110, 2011.
SOUZA, C. A. S. et al. Exigências nutricionais e crescimento de plantas de mogno (Swietenia macrophylla King.). Acta Amazônica, v.40, n.3, p.515-522, 2010.

SOUZA, P. A.; VENTURIN, N.; MACEDO, R. L. G. Adubação mineral do ipê-roxo (Tabebuia impetiginosa). Ciência Florestal, v.16, n.3, p.261-270, 2006.

SWIADER, J. M. et al. Genotypic differences in nitrate uptake and utilization efficiency in pumpkin hybrids. Journal of Plant Nutrition, v.17, p.1687-1699, 1994.

TOMAZ, M. A. et al. Eficiência de absorção, translocação e uso de cálcio, magnésio e enxofre por mudas enxertadas de Coffea arábica.

Revista Brasileira de Ciência do Solo, v.27, n.5, p. 885-892, 2003.

VENTURIN, N. et al. Adubação mineral do angicoamarelo (Peltophorum dubium (Spreng.) Taub.). Pesquisa Agropecuária Brasileira, v.34, n.3, p.441-448, 1999. 